\title{
Lie symmetries and nonlocally related systems of the continuous and discrete dispersive long waves system by geometric approach
}

Shou-Fu Tian, Tian-Tian Zhang, Pan-Li Ma, Xing-Yong Zhang

To cite this article: Shou-Fu Tian, Tian-Tian Zhang, Pan-Li Ma, Xing-Yong Zhang (2015) Lie symmetries and nonlocally related systems of the continuous and discrete dispersive long waves system by geometric approach, Journal of Nonlinear Mathematical Physics 22:2, 180-193, DOI: https://doi.org/10.1080/14029251.2015.1023562

To link to this article: https://doi.org/10.1080/14029251.2015.1023562

Published online: 04 January 2021 


\title{
Lie symmetries and nonlocally related systems of the continuous and discrete dispersive long waves system by geometric approach *
}

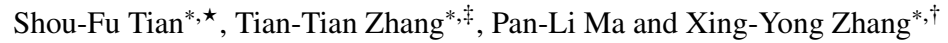 \\ Department of Mathematics and Center of Nonlinear Equations, China University of Mining and Technology, \\ Xuzhou 221116, People's Republic of China \\ ^sftian@cumt.edu.cn and shoufu2006@126.com (S.F.Tian) \\ ‡ttzhang@cumt.edu.cn and zhangtiantian_1001@126.com(T. T. Zhang) \\ ${ }^{\dagger}$ zhangxingyong163@163.com (X.Y. Zhang)
}

Received 7 July 2014

Accepted 7 January 2015

\begin{abstract}
By using the extended Harrison and Estabrook's differential forms approach, in this paper, we investigate the Lie symmetries of the continuous and discrete dispersive long waves system, respectively. Based on this method, two closed ideals written in terms of a set of differential forms are constructed for the dispersive long waves systems. Furthermore, some invariant solutions are presented for such systems. By a direct computation, it is shown that the discrete dispersive long waves system admits a Kac-Moody-Virasoro type and a Virasorolike type Lie algebra, respectively. Finally, we present an interesting relationship between the continuous case and a modified dispersive long waves system, which can be used to find nonlocal properties for such systems with each other.
\end{abstract}

Keywords: Dispersive long waves system; Lie symmetry; Conservation law; Nonlocally related systems; Geometric approach; Kac-Moody-Virasoro algebra.

2000 Mathematics Subject Classification: 35Q51, 35Q53, 35C99, 68W30, 74J35.

\section{Introduction}

As is well known, it is more and more urgent and important to investigate the integrability of nonlinear differential equations, especially, the research of symmetry property and the construction of exact solutions [4]. Nowadays, the concept of symmetries is extended from differential equations to differential-difference equations (DDEs). There exist some well-known methods, such as the intrinsic method [20], the generalized conditional symmetry method [5], the classical lie group method [1,25], the non-classical lie group method [2], and the Clarkson and Kruskal's (CK's) direct method [6]. By using Lax pairs, Lou has improved the direct method [21,22]. With the classical Lie group method, Estévez et al. [11] and Lou, Chen et al. [7, 23, 28, 29] have studied symmetry reductions of the Lax pairs for some (2+1)-dimensional equations.

In 1971, a method was proposed by Harrison and Estabrook to find the symmetries of differential equations by using a differential form technique with a geometrical flavor [13, 14]. Then, the theory is developed by Edelen of the differential form method extensively $[9,10,15]$. Recently, by

\footnotetext{
* Supported by the Fundamental Research Funds for the Central Universities under the Grant No.2013QNA41, Natural Sciences Foundation of China under the grant 11301527, and the construction project of the key discipline in universities for 12th five-year plans by Jiangsu province.

${ }^{*}$ Corresponding authors.
} 
using the discrete exterior differential technique, the method proposed by Harrison and Estabrook has been extended to study the $(2+1)$-dimensional Toda equation and discrete time Toda equation, respectively [17, 24, 27]. In this paper, we study Lie symmetries of the continuous and discrete dispersive long waves system by using the extended geometric approach.

The rest of the paper is structured as follows. In Section 2, a short summary of the exterior differential calculus is presented. In Section 3, the Lie symmetries of the continuous dispersive long waves system are obtained by virtue of the extended method. In Section 4, the method is further used to investigate the discrete dispersive long waves system. Finally, some conclusions and discussions are presented in the last section.

\section{Difference and differential form}

Following Harrison and Estabrook's work, this section we start with a short summary of the exterior differential calculus that will be useful in the rest of this paper. For a more detailed description we refer the reader to Refs. [9, 10,13-15] and [24].

Let $L$ be a lattice and $A$ be the algebra of complex valued functions on $L$. The right and left shift operators $E_{\lambda}, E_{\lambda}^{-1}$ at a node $x \in L$ in the $\lambda$-direction

$$
E_{\lambda} x=x+\hat{\lambda}, \quad E_{\lambda}^{-1} x=x-\hat{\lambda},
$$

can define a homeomorphism on the function space $A$. The homeomorphism reads

$$
E_{\lambda} f(x)=f(x+\hat{\lambda}), \quad E_{\lambda}(f(x) \cdot g(x))=E_{\lambda} f(x) \cdot E_{\lambda} g(x), \quad f, g \in A,
$$

where $\hat{\lambda}$ is the spacing in the $\lambda$-direction and the dot denotes the multiplication in $A$.

Next, the tangent space can be defined at the node $x$ of $L$ as $T L_{x}:=\operatorname{span}\left\{\left.\Delta_{\lambda}\right|_{x}, \lambda=1,2, \ldots, n\right\}$. Here $\Delta_{\lambda}$ is the differences in $\lambda$-direction defined by

$$
\Delta_{\lambda} f(x):=\left(E_{\lambda}-i d\right) f=f(x+\hat{\lambda})-f(x),
$$

where $i d$ is a identity mapping.

The dual space $T^{*} L_{x}$ of $T L_{x}$ is a space of 1-forms with a set of bases $\chi^{\lambda}$ defined on the link between $x$ and $(x+\hat{\lambda})$. The bases between $T L_{x}$ and $T^{*} L_{x}$ satisfy

$$
\chi^{\lambda}\left(\Delta_{v}\right)=\delta_{v}^{\lambda} \text { with } v \in T(L)
$$

where $\delta$ is a Kronecker function.

Let's introduce the tangent bundle and its dual over $L$

$$
T(L):=\bigcup_{x \in L} T L_{x} \text { and } T^{*}(L):=\bigcup_{x \in L} T^{*} L_{x}
$$

respectively.

Then the vectors on $T(L)$ can be defined and the whole differential algebra $\Omega^{*}=\bigoplus_{n \in Z} \Omega^{n}$ on $T^{*}(L)$ can also be constructed, where $\Omega^{n}$ is a set of $n$-forms. One can define the exterior derivative operator $d: \Omega^{k} \rightarrow \Omega^{k+1}$ as

$$
d \omega=\Delta_{\alpha} f \chi^{\alpha} \wedge \chi^{\lambda_{1}} \wedge \cdots \wedge \chi^{\lambda_{k}} \in \Omega^{k+1},
$$

where $\omega=f \chi^{\lambda_{1}} \wedge \cdots \wedge \chi^{\lambda_{k}} \in \Omega^{k}$ and $\alpha=1,2, \ldots$ 
It is easy to show that

$$
\begin{aligned}
& d^{2}=0, \\
& (d f)(v)=v(f), \quad v \in T(L), f \in \Omega^{0}, \\
& d\left(\omega \otimes \omega^{\prime}\right)=d \omega \otimes \omega^{\prime}+(-1)^{\operatorname{deg} \omega} \omega \otimes \omega^{\prime}, \quad \omega, \omega^{\prime} \in \Omega^{*},
\end{aligned}
$$

which yield the following identities

$$
\begin{aligned}
& d\left(\chi^{\lambda}\right)=0 \\
& \chi^{\lambda} \wedge \chi^{\eta}=-\chi^{\eta} \wedge \chi^{\lambda} \\
& \chi^{\lambda} f=\left(E_{\lambda} f\right) \chi^{\lambda} \text { (no summation). }
\end{aligned}
$$

From above, the discrete contraction operator $i_{V}$ is defined by [13,24,27]

$$
\left.i_{V} \omega=V\right\lrcorner \omega=\langle\omega, V\rangle, \quad V=V^{\lambda} \Delta \lambda \in T L_{x} .
$$

Thus the Lie-derivative $\mathscr{L}_{V}$ can also be introduced by

$$
\mathscr{L}_{V}=d i_{V}+i_{V} d
$$

Note that above discrete exterior differential caculus can be extended to the case of semidiscrete. For such case, the following identities should be added

$$
\begin{aligned}
& d\left(d x^{\rho}\right)=0, \\
& d x^{\rho} f=f d x^{\rho}, \\
& d x^{\rho} \wedge d x^{\eta}=-d x^{\eta} \wedge d x^{\rho}, \\
& \chi^{\mu} \wedge d x^{\rho}=-d x^{\rho} \wedge \chi^{\mu},
\end{aligned}
$$

where $x^{\rho}$ and $x^{\eta}$ are the continuous variables.

\section{Lie symmetries of the continuous dispersive long waves system}

In this section, based on the definition of Lie derivatives, which are used to find symmetries of the exterior differential equations system, we introduce isovector field and require the corresponding Lie derivative of each differential form to be a linear combination of the forms for the continuous dispersive long waves system. Compared with the discrete dispersive long waves system, in what follows, we just want to show the application of geometric methods for the continuous case. Based on that, one can further understand what happens in the discrete case.

In 1987, Boiti, Leon and Pempinelli have firstly investigated the spectral transform for a two spatial dimension extension of the dispersive long wave equation [3]. In 1988, Konopelchenko has investigated the compatibility conditions, general Bäcklund transformations and integrable equations for the two-dimensional dispersive long wave equation [18].

Now let us consider the continuous dispersive long waves system

$$
\begin{aligned}
& u_{t}=2 u u_{x}-u_{x x}+2 v_{x}, \\
& v_{y}=2 u_{x} v+2 u v_{x}+v_{x x},
\end{aligned}
$$

where $u=u(t, x, y)$ and $v=v(t, x, y)$. 
In order to convert system (3.1) to a set of differential forms, and reduce them to a first-order set by introducing two new variables given by

$$
p=u_{x}, q=v_{x}
$$

Thus system (3.1) is of the form

$$
\begin{aligned}
& u_{t}-2 u p+p_{x}-2 q=0, \\
& v_{y}-2 u q-2 v p-q_{x}=0
\end{aligned}
$$

Then we can define the following set of 3 -forms in the seven-dimensional space $N=$ $\{t, x, y, u, v, p, q\}$

$$
\begin{aligned}
& \alpha_{1}=d t \wedge d u \wedge d y-p d t \wedge d x \wedge d y \\
& \alpha_{2}=d t \wedge d v \wedge d y-q d t \wedge d x \wedge d y \\
& \beta_{1}=d u \wedge d x \wedge d y-2 u p d t \wedge d x \wedge d y-d t \wedge d y \wedge d p-2 q d t \wedge d x \wedge d y \\
& \beta_{2}=d t \wedge d x \wedge d v-2 v p d t \wedge d x \wedge d y-2 u q d t \wedge d x \wedge d y+d t \wedge d y \wedge d q
\end{aligned}
$$

and they constitute a closed ideal $\mathcal{I}_{1}$. Next an isovector field is introduced on the space $N=$ $\{t, x, y, u, v, p, q\}$

$$
V=V^{t} \partial_{t}+V^{x} \partial_{x}+V^{y} \partial_{y}+V^{u} \partial_{u}+V^{v} \partial_{v}+V^{p} \partial_{p}+V^{q} \partial_{q},
$$

where $V^{t}, V^{x}, V^{y}, V^{p}, V^{q}$ are functions with respect to $(t, x, y, u, v, p, q)$ and $V^{u}, V^{v}$ are functions with respect to $(t, x, y, u, v)$, respectively

Based on the definition of Lie-derivative in Refs. $[13,15]$, let us now consider the Lie derivatives of $\alpha_{1}$, and require them to be linear combinations of the forms system (4.2), one can obtain

$$
\left.\left.\mathscr{L}_{V} \alpha_{1}=d \alpha_{1}\right\lrcorner V+d\left(\alpha_{1}\right\lrcorner V\right)=\lambda_{1} \alpha_{1}+\lambda_{2} \alpha_{2}+\lambda_{3} \beta_{1}+\lambda_{4} \beta_{2} \in \mathcal{I}_{1}
$$

where $\lambda_{i}(i=1,2,3,4)$ are arbitrary zero-forms. No other term on the right-hand side is possible since only $\alpha_{1}, \alpha_{2}, \beta_{1}, \beta_{2}$ are the 3 -forms. Substituting (3.4), (3.5) into (3.6), we have

$$
\begin{aligned}
& V_{v}^{t}=V_{p}^{t}=V_{q}^{t}=V_{v}^{y}=V_{p}^{y}=V_{q}^{y}=0 \\
& V_{x}^{y}+p V_{u}^{y}=0 \\
& V_{q}^{u}-p V_{q}^{x}=0 \\
& V_{x}^{t}-p V_{p}^{x}+V_{p}^{u}+p V_{u}^{t}=0 \\
& V_{x}^{u}-2 p^{2} u V_{p}^{x}+2 p u V_{p}^{u}-p^{2} V_{u}^{x}-p q V_{v}^{x} \\
& \quad-2 p q V_{p}^{x}-p V_{x}^{x}+p V_{u}^{u}-q V_{v}^{u}+2 q V_{p}^{u}-V^{p}=0 .
\end{aligned}
$$

Then we consider $\mathscr{L}_{V} \alpha_{2}$ and have

$$
\left.\left.\mathscr{L}_{V} \alpha_{2}=d \alpha_{2}\right\lrcorner V+d\left(\alpha_{2}\right\lrcorner V\right) \in \mathcal{I}_{1} .
$$

It can be expressed as

$$
\mathscr{L}_{V} \alpha_{2}=\lambda_{5} \alpha_{1}+\lambda_{6} \alpha_{2}+\lambda_{7} \beta_{1}+\lambda_{8} \beta_{2}
$$


where $\lambda_{i}(i=5,6,7,8)$ are arbitrary zero-forms. Substituting (3.4), (3.5) into (3.9), one can obtain the following system

$$
\begin{aligned}
& V_{u}^{t}=V_{p}^{t}=V_{q}^{t}=V_{u}^{y}=V_{p}^{y}=V_{q}^{y}=0 \\
& V_{q}^{v}-q V_{v}^{y}-V_{x}^{y}-q V_{q}^{x}=0 \\
& V_{x}^{t}+q V_{v}^{t}=0 \\
& V^{q}+2 v p q V_{v}^{y}+2 u q^{2} V_{v}^{y}+2 v p V_{x}^{y}+2 u q V_{x}^{y}+p q V_{u}^{x} \\
& +q^{2} V_{v}^{x}+q V_{x}^{x}-p V_{u}^{v}-q V_{v}^{v}-V_{x}^{v}=0 .
\end{aligned}
$$

Then we consider $\mathscr{L}_{v} \beta_{1}$ and $\mathscr{L}_{v} \beta_{2}$ to complete the calculation, and put

$$
\begin{aligned}
& \left.\left.\mathscr{L}_{V} \beta_{1}=d \alpha_{1}\right\lrcorner V+d\left(\alpha_{1}\right\lrcorner V\right)=\xi_{1} \alpha_{1}+\xi_{2} \alpha_{2}+\xi_{3} \beta_{1}+\xi_{4} \beta_{2} \in \mathcal{I}, \\
& \left.\left.\mathscr{L}_{V} \beta_{2}=d \alpha_{1}\right\lrcorner V+d\left(\alpha_{1}\right\lrcorner V\right)=\xi_{5} \alpha_{1}+\xi_{6} \alpha_{2}+\xi_{7} \beta_{1}+\xi_{8} \beta_{2} \in \mathcal{I},
\end{aligned}
$$

where $\xi_{i}(i=1, \ldots, 6)$ are arbitrary zero-forms. We now write out the terms involving all possible basis three-forms and eliminate the multipliers. Substituting (3.4), (3.5) into (3.11), one can obtain the following systems

$$
\begin{aligned}
& V_{v}^{t}=V_{q}^{t}=V_{v}^{x}=V_{q}^{x}=0, \\
& V_{t}^{y}=V_{x}^{y}=V_{u}^{y}=V_{v}^{y}=V_{p}^{y}=V_{q}^{y}=V_{v}^{u}=V_{q}^{u}=V_{q}^{p}=0, \\
& V_{p}^{x}+V_{u}^{t}=0, \\
& V_{x}^{t}+2 u p V_{p}^{t}+2 q V_{p}^{t}-V_{p}^{u}=0, \\
& V_{u}^{u}+2 u p V_{p}^{x}+2 q V_{p}^{x}-V_{t}^{t}-V_{p}^{p}-2 u p V_{u}^{t}-2 q V_{u}^{t}+V_{x}^{x}=0, \\
& V^{q}+2 u^{2} p^{2} V_{u}^{t}+4 u p q V_{u}^{t}+u p^{2} V_{u}^{x}+u p V_{t}^{t}+2 q^{2} V_{u}^{t}-u p V_{u}^{u} \\
& \quad+p q V_{u}^{x}+p V^{u}+u V^{p}+q V_{t}^{t}-q V_{u}^{u}+\frac{1}{2} p V_{t}^{x}-\frac{1}{2} p V_{u}^{p} \\
& \quad-\frac{1}{2} q V_{v}^{p}-\frac{1}{2} V_{t}^{u}-\frac{1}{2} V_{x}^{p}=0,
\end{aligned}
$$

and

$$
\begin{aligned}
& V_{x}^{t}=V_{y}^{t}=V_{u}^{t}=V_{v}^{t}=V_{p}^{t}=V_{q}^{t}=0 \\
& V_{u}^{x}=V_{p}^{x}=V_{u}^{y}=V_{p}^{y}=V_{p}^{y}=V_{u}^{u}=V_{u}^{v}=V_{p}^{v}=V_{p}^{q}=0 \\
& V_{q}^{x}-V_{v}^{y}=0 \\
& V_{q}^{v}-2 p v V_{q}^{y}-2 u q V_{q}^{y}+V_{x}^{y}=0 \\
& V_{q}^{q}+2 p v V_{v}^{y}+2 u q V_{v}^{y}+V_{x}^{x}-V_{v}^{v}+2 v p V_{q}^{x}+2 u q V_{q}^{x}+V_{y}^{y}=0 \\
& V_{x}^{q}-4 p^{2} v^{2} V_{q}^{x}=-8 u v p q V_{q}^{x}-4 u^{2} p^{2} V_{q}^{x}+2 v p q V_{v}^{x}+2 u q^{2} V_{v}^{x}+2 p v V_{x}^{x}+2 u q V_{x}^{x} \\
& \quad-2 p v V_{q}^{q}-2 u q V_{q}^{q}+2 q V^{u}-2 v V^{p}+p V_{u}^{q}+q V_{v}^{q}+q V_{y}^{x}+2 p V^{v}+2 u V^{q}-V_{y}^{v}=0 .
\end{aligned}
$$


From systems (3.7), (3.10), (3.12) and (3.13), one can get the following infinitesimals

$$
\begin{aligned}
V^{t} & =c_{1} t+c_{3}, \\
V^{x} & =\frac{1}{2} c_{1} x+c_{5}(t+y)+c_{4}, \\
V^{y} & =c_{1} y+c_{2}, \\
V^{u} & =-\frac{1}{2} c_{1} u-\frac{1}{2} c_{5}, \\
V^{v} & =-c_{1} v, \\
V^{p} & =-c_{1} p, \\
V^{q} & =-\frac{3}{2} c_{1} q,
\end{aligned}
$$

where $c_{i}(i=1,2,3,4,5)$ are arbitrary constants.

Based on the infinitesimals (3.14), we obtain the following invariant solutions.

Solution 1: The first set of solution $u$ and $v$ is given by

$$
u=f(y), v=g(t),
$$

where $f, g$ are two arbitrary functions with respect to their arguments.

Solution 2: The second set of solution $u$ and $v$ is given by

$$
\begin{aligned}
& u=\frac{-x+2 k_{1} \sqrt{y}}{2 y+2 t}, \\
& v=\frac{k_{2}}{y+t},
\end{aligned}
$$

where $k_{1}$ and $k_{2}$ are two arbitrary constants.

Solution 3: The third set of solution $u$ and $v$ is given by

$$
\begin{aligned}
& u=\frac{\left[\left(8 k_{1}+2\right) t-x^{2}\right] M(+)+k_{2}\left[\left(16 k_{1}+4\right) t-2 x^{2}\right] U(+)-4 t\left(2 k_{1}+1\right) M(-)-k_{2} t U(-)}{2 x t\left[2 k_{2} U(+)+M(+)\right]}, \\
& v=h(t) \exp \left(\frac{\text { Numerator }}{2 k_{2} U(+)+x^{2} t M(+)^{2}}\right),
\end{aligned}
$$

with the numerator of $v$ is given by

$$
\begin{aligned}
& y\left[\left(-4 k_{2}^{2} x^{2}+8 k_{2}^{2} t+128 k_{1}^{2} k_{2}^{2} t+64 k_{1} k_{2}^{2} t\right) U(+)^{2}+\left(16 k_{1} t+32 k_{1}^{2} t-x^{2}+2 t\right) M(+)^{2}+32 k_{2}^{2} t U(-)^{2}\right. \\
& +\left(-8 t+2 x^{2}-48 k_{1} t-64 k_{1}^{2} t+4 k_{1} x^{2}\right) M(+) M(-)+32 k_{1} t\left(1+k_{1}\right) M(-)^{2}+\left(-4 k_{2} x^{2}+8 t+16 k_{2} t\right. \\
& \left.+128 k_{1}^{2} k_{2} t+64 k_{1} k_{2} t\right) M(+) U(+)+\left(16 k_{2} t-4 k_{2} x^{2}+64 k_{1} k_{2} t\right) M(+) U(-)-\left(16 k_{2} t+96 k_{1} k_{2} t\right. \\
& \left.+128 k_{1}^{2} k_{2} t\right) M(-) U(+)-32 k_{2}\left(1+2 k_{1}\right) M(-) U(-)+\left(32 k_{2}^{2} t-8 k_{2}^{2} x^{2}+128 k_{1} k_{2}^{2} t\right) U(+) U(-) \\
& \left.+4 k_{2}\left(x^{2}+2 k_{1}\right) U(+) M(-)\right],
\end{aligned}
$$


where $k_{1}, k_{2}$ are two arbitrary constants, $h(t)$ is a free function, and

$$
\begin{aligned}
& M(+)=\operatorname{KummerM}\left(-2 k_{1}+\frac{1}{2}, \frac{3}{2}, \frac{x^{2}}{4 t}\right), \\
& M(-)=\operatorname{KummerM}\left(-2 k_{1}-\frac{1}{2}, \frac{3}{2}, \frac{x^{2}}{4 t}\right), \\
& U(+)=\operatorname{KummerU}\left(-2 k_{1}+\frac{1}{2}, \frac{3}{2}, \frac{x^{2}}{4 t}\right), \\
& U(-)=\operatorname{KummerU}\left(-2 k_{1}-\frac{1}{2}, \frac{3}{2}, \frac{x^{2}}{4 t}\right) .
\end{aligned}
$$

The Kummer functions $\operatorname{KummerM}(\mu, v, z)$ and $\operatorname{KummerU}(\mu, v, z)$ solve the differential equation

$$
z U^{\prime \prime}+(v-z) U^{\prime}-\mu U=0 .
$$

By choosing the arbitrary constants $k_{1}, k_{2}$ and function $h(t)$, the simulation of the third set of solution $u$ and $v$ are shown in Figures 1 and 2.

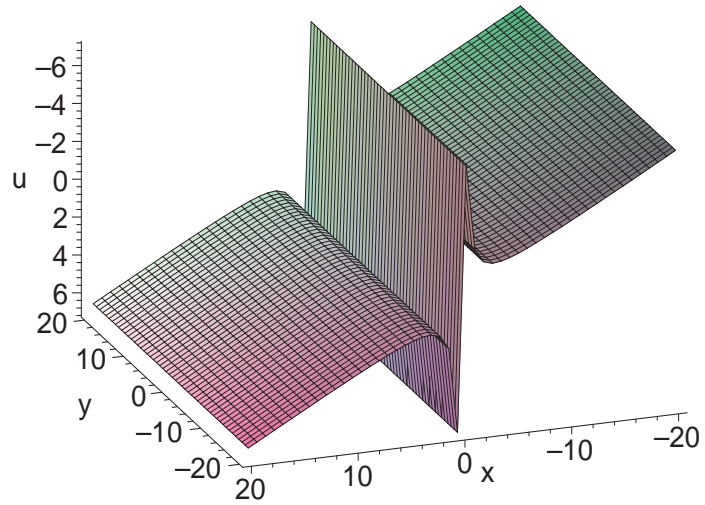

(a)

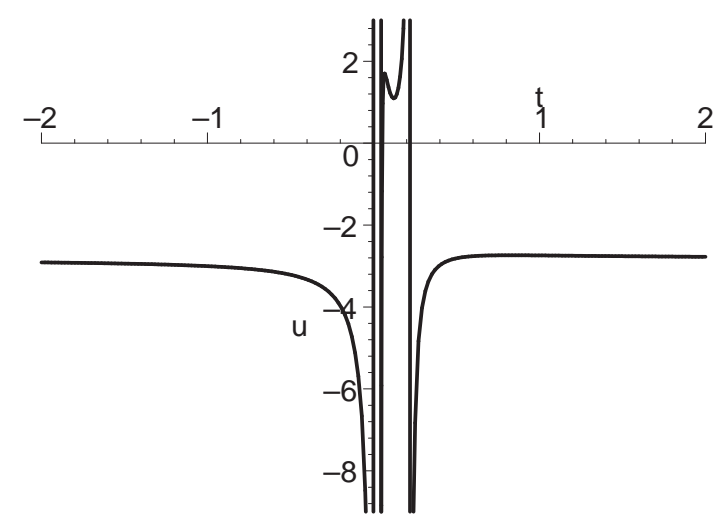

(b)

Figure 1. (Color online) The wave propagation plots of $u$ to the continuous dispersive long waves system given by (3.17), with the parameters $k_{1}=1, k_{2}=1$ and $h=1$ : (a) $t=-1$; (b) $x=-1, y=1$.

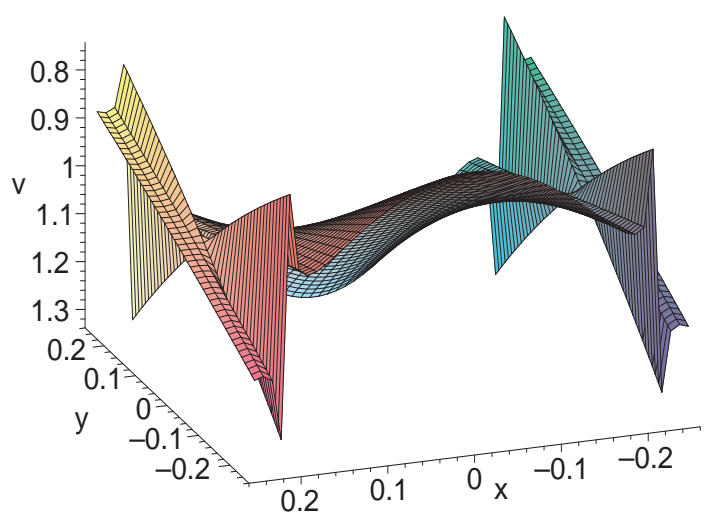

(a)

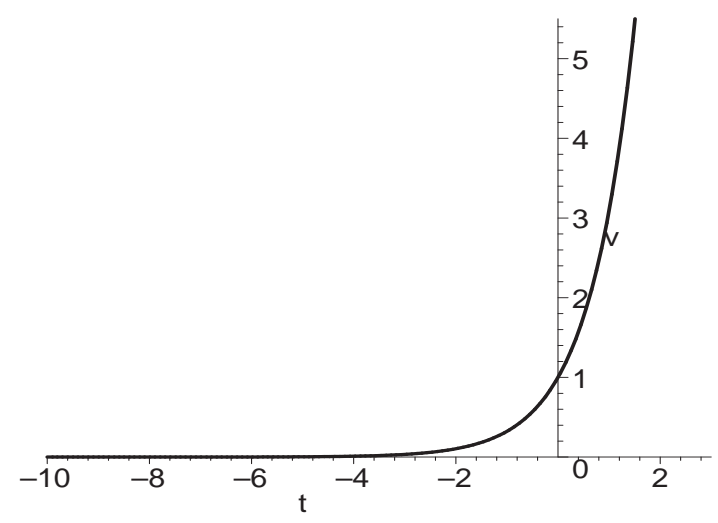

(b)

Figure 2. (Color online) The wave propagation plots of $v$ to the continuous dispersive long waves system given by (3.17), with the parameters $k_{1}=1, k_{2}=1$ and $h=1$ : (a) $t=0.01$; (b) $x=0.01, y=0.01$. 


\section{Lie symmetries of the discrete dispersive long waves system}

In what follows, based on the exterior differential system, we further introduce isovector field and require the corresponding Lie derivative of each differential form to be a linear combination of the forms for the discrete dispersive long waves system.

In 1991, Konopelchenko has firstly proposed the discrete dispersive long waves system, and investigated its Laplace transform [19]. In 1997, Shabat and Yamilov have investigated a transformation theory for the discrete dispersive long waves system [26]. Now let us consider the dispersive long waves system

$$
\begin{aligned}
& u_{n, y}=v_{n}-v_{n+1}, \\
& v_{n, x}=v_{n}\left(u_{n}-u_{n-1}\right),
\end{aligned}
$$

where $u_{n}=u_{n}(x, y)$ and $v_{n}=v_{n}(x, y)$.

Then we can define the following set of 2-forms and 3-forms in the seven-dimensional space $N=\left\{n, x, y, u_{n}, u_{n-1}, v_{n}, v_{n+1}\right\}$

$$
\begin{aligned}
& \alpha_{1}=\left[d u_{n}-\left(v_{n}-v_{n+1}\right) d y\right] \wedge \chi \wedge d x \\
& \alpha_{2}=\left[d v_{n}-\chi\left(v_{n+1}-v_{n}\right)-v_{n}\left(u_{n}-u_{n-1}\right) d x\right] \wedge d y, \\
& \beta_{1}=\left[d u_{n-1}-\chi\left(u_{n}-u_{n-1}\right)\right] \wedge d x \wedge d y, \\
& \beta_{2}=\left[d\left(v_{n+1}-v_{n}\right)+v_{n} d\left(u_{n}-u_{n-1}\right)+\left(u_{n}-u_{n-1}\right) d v_{n}\right] \wedge d x \wedge d y
\end{aligned}
$$

and they constitute a closed ideal $\mathcal{I}_{2}$. Next an isovector field is introduced on the space $N=$ $\left\{n, x, y, u_{n} \cdot u_{n-1}, v_{n}, v_{n+1}\right\}$

$$
V=V^{n} \Delta_{n}+V^{x} \partial_{x}+V^{y} \partial_{y}+V^{u_{n}} \partial_{u_{n}}+V^{u_{n-1}} \partial_{u_{n-1}}+V^{v_{n}} \partial_{v_{n}}+V^{v_{n+1}} \partial_{v_{n+1}}
$$

where $\Delta_{n} u_{n}=u_{n+1}-u_{n}$ and $\chi\left(\Delta_{n}\right)=1$.

Based on the definition of Lie-derivative in Refs. [17,24,27], let us now consider the Lie derivatives of $\alpha_{1}$, and require them to be linear combinations of the forms system (4.2), one can obtain

$$
\left.\left.\mathscr{L}_{V} \alpha_{1}=d \alpha_{1}\right\lrcorner V+d\left(\alpha_{1}\right\lrcorner V\right)=\alpha_{2} \wedge \zeta+\xi_{1} d \alpha_{2}+\xi_{2} \alpha_{1}+\xi_{3} \beta_{1}+\xi_{4} \beta_{2} \in \mathcal{I}_{2},
$$

where $\zeta$ and $\xi_{i}, i=1,2,3,4$, are arbitrary one and zero-forms. No other term on the right-hand side is possible. Substituting (4.2), (4.3) into (4.4), one has

$$
\begin{aligned}
& V_{u_{n-1}}^{n}=V_{v_{n+1}}^{n}=V_{u_{n-1}}^{x}=V_{v_{n}}^{x}=V_{v_{n+1}}^{x}=0 \\
& V_{v_{n}}^{u_{n}}+\Delta_{n} v_{n} V_{v_{n}}^{y}=0, \quad V_{v_{n+1}}^{u_{n}}+\Delta_{n} v_{n} V_{v_{n+1}}^{y}=0 \\
& v_{n} \Delta_{n} v_{n} V_{v_{n+1}}^{x}-V_{y}^{n}+\Delta_{n} v_{n} V_{u_{n}}^{n}=0 \\
& V_{u_{n-1}}^{u_{n}}+\Delta_{n} v_{n} V_{u_{n-1}}^{y}=0, \quad V_{y}^{x}-\Delta_{n} v_{n} V_{u_{n}}^{x}=0 \\
& \Delta_{n} v_{n} V_{y}^{y}+V_{y}^{u_{n}}-\Delta_{n} V^{v_{n}}-\Delta_{n} v_{n}^{2} V_{u_{n}}^{y}-\Delta_{n} v_{n} V_{u_{n}}^{u_{n}}+\left(v_{n+1}-v_{n}\right)^{2} V_{v_{n}}^{n} \\
& +\Delta_{n} u_{n-1} v_{n} v_{n+1} V_{v_{n}}^{x}-u_{n} v_{n} \Delta_{n} v_{n} V_{v_{n+1}}^{x}+u_{n-1} v_{n} \Delta_{n} v_{n} V_{v_{n+1}}^{x}+u_{n} \Delta_{n} v_{n} V_{u_{n-1}}^{n} \\
& -u_{n-1} \Delta_{n} v_{n} V_{u_{n-1}}^{n}+u_{n} v_{n+1} \Delta_{n} v_{n} V_{v_{n+1}}^{x}-u_{n-1} v_{n+1} \Delta_{n} v_{n} V_{v_{n+1}}^{x}-\Delta_{n} u_{n-1} v_{n}^{2} V_{v_{n}}^{x}=0 .
\end{aligned}
$$

Then we consider $\mathscr{L}_{V} \alpha_{2}$ and have

$$
\left.\left.\mathscr{L}_{V} \alpha_{2}=d \alpha_{2}\right\lrcorner V+d\left(\alpha_{2}\right\lrcorner V\right) \in \mathcal{I}_{2} .
$$


It can be expressed as

$$
\mathscr{L}_{V} \alpha_{2}=\xi \alpha_{2}
$$

where $\xi$ is an arbitrary zero-form. Substituting (4.2), (4.3) into (4.7), one can obtain the following system

$$
\begin{aligned}
& V_{u_{n-1}}^{y}=V_{u_{n}}^{y}=V_{v_{n+1}}^{y}=0 \\
& \Delta_{n} v_{n} V_{x}^{y}-\Delta_{n} u_{n-1} v_{n} \Delta_{n} V^{y}=0, \\
& V_{u_{n}}^{v_{n}}-\Delta_{n} u_{n-1} v_{n} V_{u_{n}}^{x}-\Delta_{n} v_{n} V_{u_{n}}^{n}=0, \\
& V_{v_{n+1}}^{v_{n}}-\Delta_{n} V_{v_{n+1}}^{n}-\Delta_{n} u_{n-1} v_{n} V_{u_{n+1}}^{x}=0 \\
& V_{u_{n-1}}^{v_{n}}-\Delta_{n} u_{n-1} v_{n} V_{u_{n-1}}^{x}-\Delta_{n} v_{n} V_{u_{n-1}}^{n}=0 \\
& \Delta_{n} V^{y}+\Delta_{n} v_{n} V_{v_{n}}^{y}=0, \Delta_{n} u_{n-1} v_{n} V_{v_{n}}^{y}+V_{x}^{y}=0 \\
& -\Delta_{n} v_{n} V_{v_{n}}^{v_{n}}+\Delta_{n} v_{n}^{2} v_{n+1} V_{v_{n}}^{n}+\Delta_{n} u_{n-1} \Delta_{n} v_{n} V_{v_{n}}^{x} \\
& -\Delta_{n} V^{v_{n}}+\Delta_{n} V^{v_{n+1}}+\Delta_{n} u_{n-1} v_{n} \Delta_{n} V^{x}+\Delta_{n} V_{n} \Delta_{n} V^{n}=0, \\
& -V_{x}^{v_{n}}-v_{n} \Delta_{n} V^{u_{n}}+\Delta_{n} v_{n} V_{x}^{n}-\Delta_{n} u_{n-1} v_{n} V_{v_{n}}^{v_{n}}+\Delta_{n} u_{n-1} v_{n} \Delta_{n} v_{n} V_{v_{n}}^{n} \\
& +\Delta_{n} u_{n-1} v_{n}^{2} V_{v_{n}}^{x}+\Delta_{n} u_{n-1} V^{v_{n}}+\Delta_{n} u_{n-1} v_{n} V_{x}^{x}=0 .
\end{aligned}
$$

Then we consider $\mathscr{L}_{v} \beta_{1}$ and $\mathscr{L}_{v} \beta_{2}$ to complete the calculation, and put

$$
\begin{aligned}
& \left.\left.\mathscr{L}_{V} \beta_{1}=d \beta_{1}\right\lrcorner V+d\left(\beta_{1}\right\lrcorner V\right)=\alpha_{2} \wedge \zeta_{1}+\lambda_{1} d \alpha_{2}+\lambda_{2} \alpha_{1}+\lambda_{3} \beta_{1}+\lambda_{4} \beta_{2} \in \mathcal{I}_{2}, \\
& \left.\left.\mathscr{L}_{V} \beta_{2}=d \beta_{2}\right\lrcorner V+d\left(\beta_{2}\right\lrcorner V\right)=\alpha_{2} \wedge \zeta_{2}+\lambda_{5} d \alpha_{2}+\lambda_{6} \alpha_{1}+\lambda_{7} \beta_{1}+\lambda_{8} \beta_{2} \in \mathcal{I}_{2},
\end{aligned}
$$

where $\zeta_{i}$ and $\xi_{j}, i=1,2, j=1, \ldots, 6$, are arbitrary one and zero-forms. We now write out the terms involving all possible basis three-forms and eliminate the multipliers. Substituting (4.2), (4.3) into (4.9), one can obtain the following systems

$$
\begin{aligned}
& V_{u_{n}}^{x}=V_{v_{n+1}}^{x}=V_{u_{n}}^{y}=V_{v_{n}}^{y}=V_{v_{n+1}}^{y}=0 \\
& \Delta_{n} V^{x}+\Delta_{n} v_{n} V_{v_{n}}^{x}+\Delta_{n} u_{n-1} V_{u_{n-1}}^{x}=0 \\
& -\Delta_{n} u_{n-1} V_{u_{n}}^{n}-\Delta_{n} u_{n-1} v_{n} V_{v_{n+1}}^{x}+V_{u_{n}}^{u_{n-1}}=0 \\
& V_{v_{n+1}}^{u_{n-1}}-\Delta_{n} u_{n-1} V_{v_{n+1}}^{n}=0, \Delta_{n} V^{y}+\Delta_{n} u_{n-1} V_{u_{n-1}}^{x}=0, \\
& \Delta_{n} u_{n-1} \Delta_{n} v_{n} V_{u_{n}}^{y}-V^{u_{n}}+\Delta_{n} u_{n-1} V_{u_{n-1}}^{u_{n-1}}-\Delta_{n} u_{n-1}^{2} V_{u_{n-1}}^{n} \\
& +\Delta_{n} v_{n} V_{v_{n}}^{u_{n-1}}-\Delta_{n} u_{n-1} u_{n-1} v_{n} V_{v_{n+1}}^{x}+\Delta_{n} u_{n} u_{n} v_{n} V_{v_{n+1}}^{x} \\
& -\Delta_{n} u_{n-1}^{2} v_{n+1} V_{v_{n+1}}^{x}-\Delta_{n} u_{n-1} v_{n+1} V_{v_{n}}^{n}+\Delta_{n} u_{n-1}^{2} v_{n+1} V_{v_{n+1}}^{x} \\
& -\Delta_{n} u_{n-1} \Delta_{n} v_{n}-\Delta_{n} u_{n-1} \Delta_{n} V^{n}+\Delta_{n} V^{u_{n-1}}=0
\end{aligned}
$$


and

$$
\begin{aligned}
& V_{x}^{y}=V_{u_{n-1}}^{y}=V_{u_{n}}^{y}=V_{v_{n+1}}^{y}=\Delta_{n} V^{y}=0, \\
& V_{u_{n-1}}^{n}+v_{n} V_{v_{n+1}}^{x}=0, \quad V_{v_{n}}^{y}+V_{v_{n+1}}^{y}=0 \text {, } \\
& v_{n} V_{v_{n+1}}^{x}-V_{u_{n}}^{n}=0, \Delta_{n} u_{n-1} V_{u_{n-1}}^{y}+v_{n} V_{v_{n}}^{y}=0 \text {, } \\
& \Delta_{n} u_{n-1} V_{u_{n}}^{y}+v_{n} V_{v_{n}}^{y}=0, \quad V_{u_{n-1}}^{x}+V_{u_{n}}^{x}=0, \quad V_{u_{n-1}}^{y}+V_{u_{n}}^{y}=0, \\
& v_{n} V_{v_{n+1}}^{u_{n}}+V_{x}^{n}-v_{n} V_{v_{n+1}}^{u_{n-1}}+\Delta_{n} u_{n-1} v_{n} V_{v_{n}}^{n}+\Delta_{n} u_{n-1} v_{n} V_{v_{n+1}}^{n}-\Delta_{n} u_{n-1}^{2} v_{n} V_{v_{n+1}}^{x}+\Delta_{n} u_{n-1} V_{v_{n+1}}^{v_{n}}=0 \text {, } \\
& -V_{u_{n-1}}^{v_{n}}+V_{u_{n-1}}^{v_{n+1}}-\Delta_{n} u_{n-1} v_{n+1} V_{u_{n-1}}^{x}+\Delta_{n} v_{n} V_{u_{n-1}}^{n}-\Delta_{n} v_{n} v_{n} V_{v_{n}}^{x}+\Delta_{n} u_{n-1} v_{n} V_{u_{n-1}}^{x}-v_{n} \Delta_{n} V^{x}=0 \text {, } \\
& V_{u_{n}}^{v_{n+1}}+v_{n} \Delta_{n} V^{x}-V_{u_{n}}^{v_{n}}+u_{n-1} \Delta_{n} v_{n} V_{u_{n}}^{x}+v_{n} \Delta_{n} v_{n} V_{v_{n}}^{x}+\Delta_{n} v_{n} V_{u_{n}}^{n}-u_{n} \Delta_{n} v_{n} V_{u_{n}}^{x}=0 \text {, } \\
& -v_{n} V_{u_{n}}^{u_{n-1}}+v_{n} V_{x}^{x}-\Delta_{n} V^{n}+\Delta_{n} u_{n-1} v_{n} V_{u_{n}}^{n}-\Delta_{n} u_{n-1}^{2} u_{n} v_{n} V_{u_{n}}^{x}+V^{v_{n}}-\Delta_{n} v_{n} v_{n} V_{v_{n+1}}^{n} \\
& +v_{n} V_{v_{n+1}}^{v_{n}}-v_{n} V_{v_{n+1}}^{v_{n+1}}+\Delta_{n} u_{n-1} V_{u_{n}}^{v_{n}}+v_{n} V_{u_{n}}^{u_{n}}+\Delta_{n} u_{n-1} v_{n} v_{n+1} V_{v_{n+1}}^{x} \\
& -\Delta_{n} v_{n} v_{n} V_{v_{n}}^{n}+\Delta_{n} u_{n-1} v_{n}^{2} V_{v_{n}}^{x}-\Delta_{n} u_{n-1} v_{n}^{2} V_{v_{n+1}}^{x}=0 \text {, } \\
& v_{n} \Delta_{n} V^{u_{n}}-v_{n} \Delta_{n} V^{u_{n-1}}+\Delta_{n} u_{n-1}^{2} v_{n}^{2} V_{v_{n+1}}^{x}-\Delta_{n} u_{n-1} v_{n} V_{v_{n}}^{v_{n+1}}+\Delta_{n} u_{n-1} v_{n} V_{v_{n+1}}^{v_{n+1}}-u_{n}^{2} v_{n} \Delta_{n} V^{x} \\
& -\Delta_{n} u_{n-1} v_{n}^{2} V_{v_{n+1}}^{n}-\Delta_{n} u_{n-1} v_{n} V_{v_{n+1}}^{v_{n}}-u_{n-1}^{2} v_{n} \Delta_{n} V^{x}+u_{n-1}^{2} v_{n} V^{x}-V_{x}^{v_{n+1}}-2 u_{n-1} u_{n} v_{n} V_{u_{n-1}}^{n} \\
& +3 u_{n-1} u_{n} v_{n} \Delta_{n} u_{n-1} V_{u_{n-1}}^{x}+\Delta_{n} u_{n-1} \Delta_{n} V^{v_{n}}+v_{n} v_{n+1} \Delta_{n} V^{y}+u_{n-1} v_{n}^{2} V_{v_{n}}^{n}-\Delta_{n} u_{n-1}^{2} v_{n}^{2} V_{v_{n}}^{x} \\
& -2 u_{n} v_{n} V_{x}^{x}+2 u_{n-1} v_{n} V_{v_{n}}^{x}+\Delta_{n} u_{n-1} \Delta_{n} v_{n} u_{n} v_{n} v_{n+1} V_{v_{n}}^{n}-2 \Delta_{n} u_{n-1}^{2} v_{n} v_{n+1} V_{v_{n+1}}^{x} \\
& -2 u_{n-1} u_{n} v_{n} V^{x}+2 \Delta_{n} u_{n-1} v_{n} v_{n+1} V_{v_{n+1}}^{n}+2 u_{n-1} u_{n} v_{n} \Delta_{n} V^{x}-\Delta_{n} v_{n} V_{x}^{n}-2 \Delta_{n} u_{n-1} V^{v_{n}} \\
& -\Delta_{n} v_{n} v_{n} V^{u_{n-1}}+\Delta_{n} v_{n} V^{u_{n}} V_{x}^{v_{n}}-\Delta_{n} u_{n-1} v_{n} V_{u_{n-1}}^{u_{n-1}}+u_{n}^{2} v_{n} V_{u_{n-1}}^{n}+u_{n-1}^{2} v_{n} V_{u_{n-1}}^{n}-u_{n}^{3} v_{n} V_{u_{n-1}}^{x} \\
& +\Delta_{n} u_{n-1} v_{n} V_{u_{n-1}}^{u_{n}}+u_{n-1}^{3} v_{n} V_{u_{n-1}}^{x}+\Delta_{n} u_{n-1} v_{n+1} V_{v_{n+1}}^{v_{n}}-v_{n} \Delta_{n} v_{n} V_{v_{n}}^{u_{n-1}}+2 \Delta_{n} u_{n-1} v_{n} \Delta_{n} V^{n} \\
& -\Delta_{n} u_{n-1} v_{n+1} \Delta_{n} V^{n}+\Delta_{n} u_{n+1}^{2} v_{n+1}^{2} V_{v_{n+1}}^{x}-\Delta_{n} u_{n-1} v_{n+1}^{2} V_{v_{n}}^{n}-\Delta_{n} u_{n-1} v_{n+1}^{2} V_{v_{n+1}}^{n}+v_{n} v_{n+1} V_{v_{n}}^{u_{n}} \\
& -\Delta_{n} u_{n-1} v_{n+1} V_{v_{n+1}}^{v_{n+1}}-\Delta_{n} u_{n-1} v_{n+1} V_{v_{n+1}}^{v_{n+1}}+\Delta_{n} u_{n-1} v_{n+1} V_{v_{n}}^{v_{n}}-v_{n}^{2} \Delta_{n} V^{y}+\Delta_{n} u_{n-1}^{2} V_{u_{n-1}}^{v_{n}} \\
& -v_{n}^{2} V_{v_{n}}^{u_{n}}=0 \text {. }
\end{aligned}
$$

Solving these systems (4.5), (4.8), (4.10) and (4.11), one can obtain

$$
\begin{aligned}
& V^{n}=0, \\
& V^{x}=f(x), \\
& V^{y}=g(y), \\
& V^{u_{n}}=h(x)+k(y) e^{f(x)}+n f^{\prime}(x), \\
& V^{u_{n-1}}=h(x)+k(y) e^{f(x)}+(n-1) f^{\prime}(x), \\
& V^{v_{n}}=g(y) e^{f(x)}-n k^{\prime}(y) e^{f(x)}, \\
& V^{v_{n+1}}=g(y) e^{f(x)}-(n+1) k^{\prime}(y) e^{f(x),}
\end{aligned}
$$

where $f(x), g(y), h(x)$ and $k(y)$ are arbitrary functions and the primes denote derivatives with respect to the respective arguments.

On the one hand, based on the infinitesimals (4.12), we obtain the following invariant solutions 
Solution 1: Taking $f=0, g=y, h=x$ and $k=y$, one can obtain the first set of solution $u_{n}$ and $v_{n}$ given by

$$
\begin{aligned}
& u_{n}=F_{1}(x)+F_{2}(y), \\
& v_{n}=-n F_{2}^{\prime}(y),
\end{aligned}
$$

where $F_{1}(x)$ and $F_{2}(y)$ are two continuously differentiable functions with respective to $x$ and $y$, respectively.

Solution 2: Taking $f=1, g=y, h=x$ and $k=y$, one can obtain the first set of solution $u_{n}$ and $v_{n}$ given by

$$
\begin{aligned}
& u_{n}=G_{1}(x)+d_{1} \ln y+d_{2} \ln \left[y G_{2}(x)\right], \\
& v_{n}=G_{3}(y)-\frac{n\left(d_{1}+d_{2}\right)}{y},
\end{aligned}
$$

where $d_{1}, d_{2}$ are two arbitrary constants, $G_{1}(x), G_{2}(x)$ and $G_{3}(y)$ are three continuously differentiable functions with respective to $x$ and $y$, respectively.

Solution 3: Taking $f=-1, g=y, h=x$ and $k=y$, one can obtain the first set of solution $u_{n}$ and $v_{n}$ given by

$$
\begin{aligned}
& u_{n}=H_{1}(x)+\widetilde{d}_{1} \ln y+\widetilde{d}_{2} \ln \left[y H_{2}(x)\right], \\
& v_{n}=H_{3}(y)-\frac{n\left(\widetilde{d}_{1}+\widetilde{d}_{2}\right)}{y},
\end{aligned}
$$

where $\widetilde{d}_{1}, \widetilde{d}_{2}$ are two arbitrary constants, $H_{1}(x), H_{2}(x)$ and $H_{3}(y)$ are three continuously differentiable functions with respective to $x$ and $y$, respectively.

On the other hand, from (4.12), one can obtain the bases of the symmetry algebra

$$
\begin{aligned}
X(f)= & f(x) \partial_{x}+k(y) e^{f(x)} \partial_{u_{n}}+n f^{\prime}(x) \partial_{u_{n}}+k(y) e^{f(x)} \partial_{u_{n-1}}+(n-1) f^{\prime}(x) \partial_{u_{n-1}}+g(y) e^{f(x)} \partial_{v_{n}} \\
& -n k^{\prime}(y) e^{f(x)} \partial_{v_{n}}+g(y) e^{f(x)} \partial_{v_{n+1}}-(n+1) k^{\prime}(y) e^{f(x)} \partial_{v_{n+1}}, \\
Y(g)= & g(y) \partial_{y}+g(y) e^{f(x)} \partial_{v_{n}}+g(y) e^{f(x)} \partial_{v_{n+1}}, \\
U(h)= & h(x) \partial_{u_{n}}+h(x) \partial_{u_{n-1}}, \\
V(k)= & k(y) e^{f(x)} \partial_{u_{n}}+k(y) e^{f(x)} \partial_{u_{n-1}}-n k^{\prime}(y) e^{f(x)} \partial_{v_{n}}-(n+1) k^{\prime}(y) e^{f(x)} \partial_{v_{n+1}} .
\end{aligned}
$$

By a direct computation, one can obtain the associated Kac-Moody-Virasoro type Lie algebra between these vector fields:

$$
\begin{aligned}
& {\left[X\left(f_{1}\right), X\left(f_{2}\right)\right]=X\left(f_{1} f_{2}^{\prime}-f_{1}^{\prime} f_{2}\right),} \\
& {\left[Y\left(g_{1}\right), Y\left(g_{2}\right)\right]=Y\left(g_{1} g_{2}^{\prime}-g_{1}^{\prime} g_{2}\right),} \\
& {[X(f), U(h)]=U\left(f h^{\prime}\right),} \\
& {[X(f), V(k)]=V\left(f h^{\prime}\right),} \\
& {[Y(g), U(h)]=U\left(g h^{\prime}\right),} \\
& {[Y(g), V(k)]=V\left(f k^{\prime}\right) .}
\end{aligned}
$$


In order to find an exactly Virasoro-like algebra by choosing appropriately the functions $f(x), g(y), h(x)$ and $k(y)$, one can further investigate the brackets appearing in (4.17). According to the computation given by Heredero and Reyes [16], we obtain a Virasoro-like algebra for the vector field $Y(g)$. For arbitrary functions $g_{i}$ as $g_{i}=g_{i}(y)$, by taking $g_{j}=\mathrm{i} g_{i} \tan (\mathrm{i} y+d)(i \neq j)$, one obtains

$$
\left[Y\left(g_{i}\right), Y\left(g_{j}\right)\right]=\left(g_{i}-g_{j}\right) Y\left(g_{i}+g_{j}\right), i, j=1,2, \ldots,
$$

where $d$ is an arbitrary constant. Based on the Ref. [16], one can show that the vector fields $Y\left(g_{i}\right)$ $(i=1,2, \ldots)$ about the brackets $(4.18)$ generate a Virasoro-like algebra.

\section{Conclusions and remarks}

For the continuous dispersive long waves system (3.1), there is an interesting relation to published work. Let $u$ and $v$ satisfy the following transformation

$$
\begin{aligned}
& u=q+r, \\
& v_{x}=r_{x x}-q m_{x}+r m_{x}-q q_{x}-q r_{x}-r q_{x}-r r_{x},
\end{aligned}
$$

and $m$ satisfies

$$
m_{y}=-q r
$$

system (3.1) can be reduced to the following system

$$
\begin{aligned}
& q_{t}+r_{t}=-q_{x x}-2 q m_{x}+r_{x x}+2 r m_{x}, \\
& m_{y}+q r=0,
\end{aligned}
$$

where $q, r$ and $m$ are functions with respective to $t, x$ and $y$. Considering the system (5.3), one can obtain the subsystem given by

$$
\begin{aligned}
& q_{t}+q_{x x}+2 q m_{x}=0, \\
& r_{t}-r_{x x}-2 r m_{x}=0, \\
& m_{y}+q r=0,
\end{aligned}
$$

which is called (2+1)-dimensional modified generalized long dispersive wave system [12]. It is value mentioning that the system (3.1) is a nonlocally related PDE system for the system (5.4). By considering the local properties of systems (3.1) and (5.4), respectively, one can further investigate the nonlocal properties for such systems with each other.

Recently, Chen, etal [8]. investigate nonlocal symmetry of system (5.4) and its applications by virtue of its eigenfunctions in Lax pairs. By applying the general Lie symmetry approach, they obtain the finite symmetry transformation and similarity reductions to present explicit solitoncnoidal wave solution, which can be reduced to the two-dark-soliton solution in one special case.

In this paper, based on an effective extended geometric approach, we obtain the Lie symmetries of the continuous and discrete dispersive long waves systems (3.1) and (4.1), respectively. Moreover, based on a direct computation, we obtain a Kac- Moody-Virasoro type and a Virasoro-like type Lie algebra from the discrete case, respectively. Finally, we construct a relationship between system (3.1) and (5.4). The relationship yields to a nonlocally related PDE system for the continuous case, 
which can be used to find nonlocal symmetries for the dispersive long waves system. By virtue of the inherently geometrical nature of forms, one can further study some geometrical insight into the process. The paper shows that the extended geometric approach provides a direct and more powerful mathematical tool to investigate nonlinear differential-difference equations in mathematical physics.

\section{Acknowledgments}

The authors are grateful to the anonymous referees very detailed comments, corrections and pointing out some of the references. He/She gave much work on this paper, which enhances this paper in several respects. In some sense, he/she is a joint author of this paper. This work is supported by the Fundamental Research Funds for the Central Universities under the Grant No. 2013QNA41, Natural Sciences Foundation of China under the grant 11301527, and the construction project of the key discipline in universities for 12th five-year plans by Jiangsu province.

\section{References}

[1] G. W. Bluman and S. Kumei, Symmetries and differential equations, New York: Springer, 1989.

[2] G. W. Bluman and J. D. Cole, The general similarity solution of the heat equation, J. Math. Mech. 18 (1969) 1025-1042.

[3] M. Boiti, J. J. P. Leon and F. Pempinelli, Spectral transform for a two spatial dimension extension of the dispersive long wave equation, Inverse Problems 3 (1987) 371-387.

[4] B. J. Cantwell, Introduction to Symmetry Analysis, Cambridge Texts in Applied Mathematics, CUP, 2002.

[5] K. S. Chou and C. Z. Qu, Generalized conditional symmetries of nonlinear differential-difference equations, Phys. Lett. A 280 (2001) 303-308.

[6] P. A. Clarkson and M. D. Kruskal, New similarity solutions of the Boussinesq equation, J. Math. Phys. 30 (1989) 2201-2213.

[7] Y. Chen and Z. Z. Dong, Symmetry reduction and exact solutions of the generalized Nizhnik-NovikovVeselov equation, Nonlinear Anal. 71 (2009) 810-817.

[8] J. C. Chen and Y. Chen, Nonlocal symmetry constraints and exact interaction solutions of the $(2+1)$ dimensional modified generalized long dispersive wave equation, J. Nonlinear Math. Phys., 21 (2014) 454-472

[9] D. G. B. Edelen, Applied Exterior Calculus (John Wiley and Sons, New York, 1985).

[10] D. G. B. Edelen, Order-independent method of characteristics, Internat. J. Theoret. Phys. 28 (1989) 303-333.

[11] P. G. Estévez, M. L. Gandarias and J. Prada, Symmetry reductions of a 2+1 Lax pair, Phys. Lett. A 343 (2005) 40-47.

[12] P. G. Estévez, Darboux transformation and solutions for an equation in $2+1$ dimensions, J. Math. Phys. 40 (1999) 1406-1419.

[13] B. K. Harrison and F. B. Estabrook, Geometric approach to invariance groups and solution of partial differential systems, J. Math. Phys. 12 (1971) 653-666.

[14] B. K. Harrison, Differential form symmetry analysis of two equations cited by Fushchych, in Proc. 2nd Int. Conf. Symmetry in Nonlinear Mathematical Physics, Vol. 1, eds. M. I. Shkil, A. G. Nikitin and V. M. Boyko (Ukrainian, July 1997), pp. 21-33.

[15] B. K. Harrison, The differential form method for finding symmetries, SIGMA 1 (2005) 1-12.

[16] R. Hernandez Heredero and E. G. Reyes, Geometric Integrability of the Camassa-Holm Equation. II, Int. Math. Res. Notices 2012 (2012) 3089-3125.

[17] X. Y. Jia and N. Wang, Geometric approach to Lie symmetry of discrete time Toda equation, Chin. Phys. Lett. 26(8) (2009) 1 (3pp).

[18] B. G. Konopelchenko, The two-dimensional second-order differential spectral problem: compatibility conditions, general BTs and integrable equations, Inverse Problems 4 (1988) 151-163. 
[19] B. G. Konopelchenko, The nonabelian 1+1-dimensional Toda lattice as the periodic fixed point of the Laplace transform for the 2+1-dimensional integrable system, Phys. Lett. A 156 (1991) 221-222.

[20] D. Levi and P. Winternitz, Continuous symmetries of discrete equations, Phys. Lett. A 152 (1991) 335338.

[21] S. Y. Lou and H. C. Ma, Non-Lie symmetry groups of (2+1)-dimensional nonlinear systems obtained from a simple direct method, J. Phys. A: Math. Gen. 38 (2005) L129-L137.

[22] S. Y. Lou and X. Y. Tang, Nonlinear methods of mathematical physics, science and technology, Bei Jing, 2006.

[23] S. Y. Lou, X. R. Hu and Y. Chen, Nonlocal symmetries related to Bäcklund transformation and their applications, J. Phys. A: Math. Theor. 45 (2012) 155209 (14pp).

[24] H. J. Li, D. S. Wang, S. K. Wang, K. Wu and W. Z. Zhao, On geometric approach to Lie symmetries of differential-difference equations, Phys. Lett. A 372 (2008) 5878-5882.

[25] P. J. Olver, Application of Lie group to differential equations, Berlin: Springer; 1986.

[26] A. B. Shabat and R. I. Yamilov, To a transformation theory of two-dimensional integrable systems, Phys. Lett. A 227 (1997) 15-23.

[27] K. Wu, W. Z. Zhao and H. Y. Guo, Difference discrete connection and curvature on cubic lattice, Sci. China Ser. A: Math. 49 (2006) 1458-1476.

[28] X. P. Xin and Y. Chen, A method to construct the nonlocal symmetries of nonlinear evolution equations, Chin. Phys. Lett. 30 (2013) 100202 (4pp).

[29] H. Y. Zhi, Symmetry reductions of a Lax pair for (2+1)-dimensional differential Sawada-Kotera equation, Commun. Theor. Phys. 51 (2009) 777-780. 